\title{
A New Control Approach for Switching Shunt Damping
}

\author{
Dominik Niederberger ${ }^{a},{\text { Manfred } \text { Morari }^{a} \text { and Stanislaw Pietrzko }}^{b}$ \\ ${ }^{a}$ Automatic Control Laboratory, ETH - Swiss Federal Institute of Technology, Zurich, \\ Switzerland \\ ${ }^{b}$ EMPA - Swiss Federal Laboratories for Materials Testing and Research, Dubendorf, \\ Switzerland
}

\begin{abstract}
This paper presents a new control approach for piezoelectric switching shunt damping. Recently, semi-active controllers have been used to switch piezoelectric materials in order to damp vibration. These switching shunt circuits allow a small implementation and require only little power. However, the control laws to switch these shunts are derived heuristically and therefore it remains unclear, if a better control law for a given shunt topology exists. We present a new control approach based on the Hybrid System Framework. This allows the modelling of the switched composite system as a hybrid system. Once the hybrid system description is obtained, a receding horizon optimal control problem can be solved in order to get the optimal switching sequence. As the computation time to solve this optimization problem is too long for real-time applications, we will show that the problem can be solved off-line and the solution stored in a look-up table. This allows a real-time implementation of the switch controller. Moreover, control rules can be derived from this look-up table, and we will demonstrate that in some situations the controllers proposed in previous papers generate near optimal switching. In this paper, we will investigate two shunt topologies with switches and compare the performance between the heuristically derived control laws and the optimal new control laws. Simulations and experiments show the improvement with the new controllers. This is very promising, since this new control approach can be applied for more complex shunt circuits with many switches, where the derivation of a heuristic switching law would be very difficult.
\end{abstract}

Keywords: Active Vibration Control, Shunt Damping, State-Switching, Hybrid Systems, Optimal Control, Piezoelectric

\section{INTRODUCTION}

In the past, vibration of mechanical structures has been suppressed using passive damping materials, which are not very effective for low frequency vibration and add much additional weight to the structure. Therefore active vibration control ${ }^{1-4}$ was introduced that can damp low frequencies effectively but needs many electronic devices and much external energy making this technology expensive. Shunt damping ${ }^{5-7}$ is a new approach, where an electrical circuit is connected to the terminals of a piezoelectric patch. It was shown that a resonant shunt consisting of a resistor and inductor $\left(R-L\right.$ shunt $\left.{ }^{5}\right)$, achieves very good vibration suppression for one structure resonance. However, this shunt suffers from the drawback that it is very sensitive to parameter variations, such as temperature, structural load or piezoelectric parameter variations. This means that if the $R-L$ shunt gets de-tuned due to environmental changes, the damping performance will suffer. Therefore, adaptive $R-L$ shunts ${ }^{8,9}$ were proposed that are able to tune online. However, $R-L$ shunts still have the drawback that very high inductor values are required (up to a few thousand Henrys) and this can only be implemented with active electronic components. Therefore, simpler shunts are needed that are less expensive to implement and require no power supply. One solution that may fulfill these demands is the use of switching shunts, like those in Figure 1. These shunts with switches are very easy to implement, as the switches can be realized with simple transistors. Thus the complexity of the circuits can be kept to a minimum since no bulky or virtual inductors are required. In the past, switching shunts have been proposed by Clark and Corr ${ }^{10-13}$ and Richard et al. ${ }^{14,15}$, but the problem of the optimal switching sequence and optimal switching shunt topology has not been solved yet. We will address this problem and apply the Hybrid System Framework ${ }^{16-19}$ to obtain the optimal switching sequence.

Send correspondence to D.Niederberger: E-mail: niederberger@control.ee.ethz.ch, Telephone: ++41 16327163 , Address: Automatic Control Laboratory, Swiss Federal Institute of Technology, Physikstr. 3, CH-8092 Zurich, Switzerland 


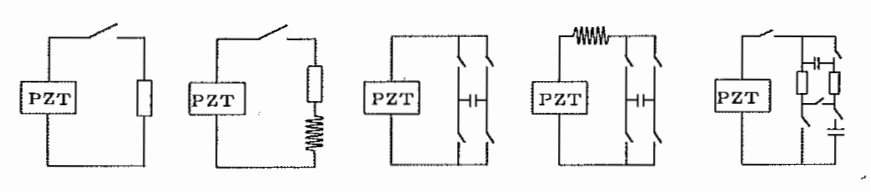

Figure 1. Shunt topologies

This paper is presented in the following sections. In Section 2, the modelling of shunted piezoelectric composite structures is shown. Section 3 reviews the heuristic controllers for switching $R$ and $R-L$ shunts. Then in Section 4, we introduce the Hybrid System Framework and demonstrate how to calculate the optimal switching sequence for different switching shunts. Simulation and experimental results in Section 5 and 6 are followed by conclusions in Section 7.

\section{ELECTRICAL EQUIVALENT MODEL}

Composite structures with an electrically shunted piezoelectric transducer can be modelled as an electrical equivalent system ${ }^{8}$. In Figure 2, the electrical equivalent circuit for one structural mode with a shunted piezoelectric patch is shown. The state-space representation of this system is

$$
\begin{aligned}
& \dot{x}=\left[\begin{array}{ccc}
0 & 1 / C_{m i} & 0 \\
-1 / L_{m i} & -R_{m i} / L_{m i} & -1 / L_{m i} \\
0 & 1 / C_{p} & -1 /\left(C_{p} Z\right)
\end{array}\right] x+\left[\begin{array}{c}
0 \\
1 / L_{m i} \\
0
\end{array}\right] P_{i} \\
& y=x,
\end{aligned}
$$

with the state vector

$$
x=\left[U_{c}, \nu, U_{p}\right]^{T},
$$

where $\nu$ denotes the velocity and $P_{i}, R_{m i}, L_{m i}, C_{m i}, C_{p}$, and $Z$ the corresponding disturbance force, the equivalent damping, mass, stiffness, piezoelectric capacitance and the shunt impedance. For more details, the reader is referred to Niederberger et. al ${ }^{8}$.

The transfer function of this electrical equivalent system is equal to other models considered for shunt damp$\operatorname{ing}^{5,10,11}$. However, the electrical equivalent model allows easy simulations of the shunted composite structure in electronic simulators like Saber* or PSpice ${ }^{\dagger}$. We will consider this model for the optimal control design in Section 4 that minimizes the vibration, i.e.

$$
\min _{S} \int_{0}^{\infty} \nu(t)^{2} d t
$$

where the disturbance force $P_{i}$ is a Dirac-impulse.

${ }^{*}$ SaberSketch and SaberScope, Version 2.4, Avant! Corp., 9205 S.W. Gemini Drive, Beaverton, Or 97008.
${ }^{\dagger}$ PSpice Schematics, Evaluation Version 9.1, Cadence Design Systems, www.cadence.com

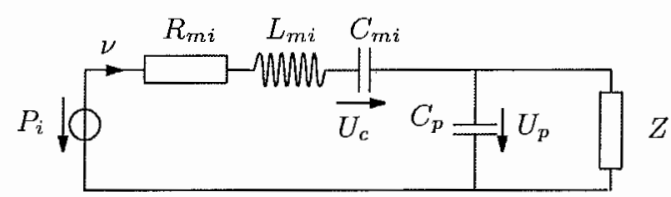

Figure 2. Electrical equivalent model of the piezoelectric composite structure. 


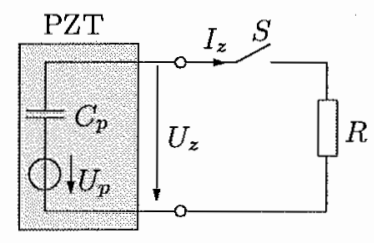

a)

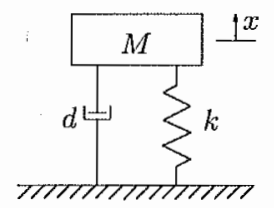

b)

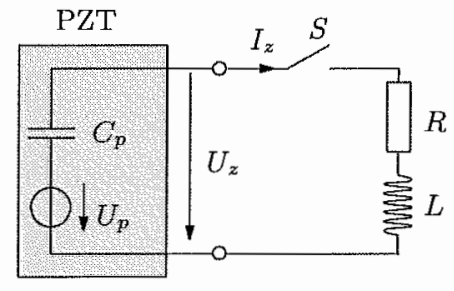

c)

Figure 3. a) Switching $R$ Shunt, b) Single degree of freedom (SDOF) system whose stiffness can either be $k=k_{0}+\Delta k$ or $k=k_{0}$.

\section{HEURISTIC CONTROL APPROACHES}

In the following, we present some heuristic control laws for switching shunt damping, where the switching shunt comprises either a switched $R$ or $R-L$, network.

\subsection{Switching R-Shunt - The State-Switching and SSD Technique}

Clark et al. ${ }^{10,11}$ and Richard et al. ${ }^{14}$ proposed controllers for the switching $R$ shunt that is shown in Figure 3 a). Depending on the switch state $S$, a resistor $R$ is shunted to the piezoelectric transducer in order to damp vibration.

\subsubsection{State-Switching}

Originally, Larson ${ }^{20}$ coined the expression "State-Switching". By switching a piezoelectric transducer between open and short-circuit states, an acoustic driver's stiffness (and therefore its natural frequency) were changed in order to track a changing frequency with high amplitude. Based on this work, Clark et al. ${ }^{10,11}$ introduced the State-Switching technique for shunt damping. First, the switch controller was derived for a mechanical single degree of freedom $(S D O F)$ system like in Figure $3 \mathrm{~b}$ ). The aim is to suppress the vibration of mass $M$, that is attached by a variable spring with either $k=k_{0}$ or $k=k_{0}+\Delta k$ where $\Delta k>0$. Additionally, the mass is damped by a damper $d$. The mass $M$ is subjected to a tonal excitation force with a frequency equal to the resonance frequency of the system. The idea of State Switching is that the mass starts off in the high-stiffness $\left(k=k_{0}+\Delta k\right)$. When the mass reaches the maximum displacement, the potential energy is also at a maximum, defined by

$$
E_{\text {pot }}^{m a x}=\frac{1}{2} \underbrace{\left(k_{0}+\Delta k\right)}_{k} x_{m a x}^{2} .
$$

At this point, the stiffness is switched to a low stiffness state $\left(k=k_{0}\right)$ such that the potential energy is

$$
E_{\text {pot }}^{\text {max }}=\frac{1}{2} k_{0} x_{\text {max }}^{2}
$$

which is less than before. The difference in energy is

$$
\Delta E=\frac{1}{2}(\Delta k) x_{\max }^{2}
$$

This energy is released from the system, when switching occurs. The spring is kept in the low stiffness state until the modal displacement returns to equilibrium (i.e. $x(t)=0$ ). Then the spring is switched back to the high stiffness state and the entire cycle repeats. The control law for the stiffness $k$ can be expressed as

$$
k(t)= \begin{cases}k_{0}+\Delta k & : \quad x(t) \cdot \dot{x}(t) \geq 0 \\ k_{0} & : \quad x(t) \cdot \dot{x}(t)<0\end{cases}
$$


This heuristic control law was then applied for shunted piezoelectric structures, because one structural mode of the piezoelectric composite structure can be modelied as a SDOF system and the corresponding stiffness is changed by the piezoelectric actuator between its open and closed-circuit states by approximately $1 /\left(1-k_{i j}\right)$, where $k_{i j}$ is the appropriate piezoelectric coupling factor. The control law of the shunt impedance is then

$$
Z(t)=\left\{\begin{array}{ccc}
\infty \Omega & \text { if } & x(t) \cdot \dot{x}(t) \geq 0 \\
0 \Omega & \text { if } & x(t) \cdot \dot{x}(t)<0
\end{array}\right.
$$

or the control law for the ideal switch in Figure 3 a) with $R=0 \Omega$ can be expressed as

$$
S(t)=\left\{\begin{array}{lll}
0 & \text { if } & x(t) \cdot \dot{x}(t) \geq 0 \\
1 & \text { if } \quad x(t) \cdot \dot{x}(t)<0
\end{array}\right.
$$

In real applications, the impedance $R$ of the switch cannot be $0 \Omega$. Clark et al. ${ }^{11}$ also performed an $R$-parameter study, where the switching law remained the same for different $R$ values. In this study, the optimal $R$ for their system was around $0.18 \mathrm{~m} \Omega$. However, it remains unclear, if the State Switching technique is the optimal switching law, especially for $R \neq 0$, because this was not taken into account in the derivation of the StateSwitching law.

\subsubsection{Synchronized Switch Damping - SSD}

Whereas the State Switching law keeps the piezoelectric element in each of the high- and low-stiffness states for one quarter-cycle increments, the synchronized switch damping (SSD) by Richard et al. ${ }^{14}$ holds the actuator in the high-stiffness state for most of the vibration cycle, but is momentarily pulsed to the low-stiffness state to dissipate stored energy. The switch is intermittently shut to discharge the piezoelectric capacitance $C_{p}$ each time the strain reaches a maximum. Afterwards, the switch is reopened in order to allow the voltage to further develop across the electrodes.

\subsection{Switching R-L Shunt - The SSDI Technique}

Switching $R-L$ shunts are shown in Figure $3 \mathrm{c}$ ). Depending on the switch state $S$, an $R-L$ network is connected to the terminals of a piezoelectric transducers with the view of minimizing the vibration of the structure. The synchronized switch damping inductor (SSDI) ${ }^{13,15}$ technique is based on the SSD methodology. It says that if the product of the velocity $\nu(t)$ and $U_{z}$ becomes greater than zero, the switch is shut. The switch is opened again, when the applied charge reaches a peak with the opposite sign from where it began (about $1 / 2$ of the time period of the $C_{p}-L$ resonance). In the SSDI technique, the optimal value of $L$ is more than 20 times smaller than for the standard $R-L$ shunt. Therefore, virtual or synthetic inductors may not be required. Recently, Corr and Clark $^{13}$ presented switched $R-L$ shunts for multi-mode vibration suppression.

\section{HYBRID SYSTEM FRAMEWORK APPROACH}

In this section, we show how to calculate the optimal switching sequence for the switching shunt controller. First, the structure with the switching shunt is modelled as a Hybrid System. Once the hybrid system description is obtained, a Receding Horizon Optimal Control (RHOC) methodology is used to get the optimal switching sequence. As the online calculation for the RHOC-methodology takes too much time for real time implementation, an optimal feedback solution approach will be investigated. We will show that the first approximation of this optimal feedback solution for the switching $R$ shunt is the heuristic State-Switching controller ${ }^{10,11}$. For the switching $R-L$ shunt, we will demonstrate that based on the RHOC-methodology the switching can be improved by a simple modification of the SSDI technique.

\subsection{Hybrid System Modelling}

The switching shunted piezoelectric transducer bonded to a mechanical structure can be modelled as a Hybrid System $^{16,17,19}$, since it comprises continuous and discrete parts. For the simple case of one switch with open 
admittance of 0 and closed admittance of $1 / Z_{\text {closed }}$, the system has two different dynamics depending on the switch state $S$. For example, the switching $R$ shunt composite system can be formulated as

$$
\begin{aligned}
\dot{x}(t) & =\left\{\begin{array}{lll}
A_{1} x(t) & \text { if } & S(t)=0 \\
A_{2} x(t) & \text { if } & S(t)=1
\end{array}\right. \\
y(t) & =x(t)
\end{aligned}
$$

where $x=\left[U_{c}(t), \nu(t), U_{p}(t)\right]^{T}$ and

$$
A_{1}=\left[\begin{array}{ccc}
0 & 1 / C_{i} & 0 \\
-1 / L_{i} & -R_{i} / L_{i} & -1 / L_{i} \\
0 & 1 / C_{p} & 0
\end{array}\right] \text { and } A_{2}=\left[\begin{array}{ccc}
0 & 1 / C_{i} & 0 \\
-1 / L_{i} & -R_{i} / L_{i} & -1 / L_{i} \\
0 & 1 / C_{p} & -1 /\left(C_{p} Z_{\text {closed }}\right) .
\end{array}\right]
$$

The Dirac-impulse in $P_{i}(t)$ is translated into the systems's initial state $x_{0}$. The parameters of the system can be found in Table 1, where the system is designed to have one resonance at $1 \mathrm{~Hz}$. Notice that there is a fourth state $I_{L}$, if the switching $R-L$ network is considered.

\subsection{Receding Horizon Optimal Control}

By taking into account (10)-(12) we can formulate the following discrete time version of the optimal control problem (3)

$$
\begin{aligned}
& \min _{\left[S_{t}(0), \ldots, S_{t}\left(N_{s}-1\right)\right]} \sum_{k=0}^{N_{s}-1} v(k)^{2} \\
& \text { subj. to }\left\{\begin{array} { l } 
{ x ( 0 ) = x ( t ) } \\
{ S _ { t } ( k ) } \\
{ x ( k + 1 ) }
\end{array} \quad \left[\begin{array}{lll}
A_{1}^{d} x(k) & \text { if } & S_{t}(k)=0 \\
A_{2}^{d} x(k) & \text { if } & S_{t}(k)=1 \\
v(k) & = & {\left[\begin{array}{lll}
0 & 1 & 0
\end{array}\right] x(k),}
\end{array}\right.\right.
\end{aligned}
$$

where $N_{s}$ denotes prediction horizon, and matrices $A_{1}^{d}, A_{2}^{d}$ are obtained by discretizing the continuous time model (10)-(12) with the sampling time $T_{s}$. Note that in continuous time optimization was carried out for the infinite time (3) while here the horizon $N_{s}$ is finite. In practical applications $N_{s}$ is chosen to be sufficiently large to capture the transient time of the system (hence reducing discrepancy between the continuous time optimization and its discrete time counterpart) and yet small enough as not to make the overall problem intractable.

Solution to the optimal control problem (13) is an optimal switching sequence $S_{t}^{*}(0), \ldots, S_{t}^{*}\left(N_{s}-1\right)$, where subscript $t$ denotes that this optimal switching sequence depends on the current state $x(t)$, i.e., it is different for every time instant $t$. On its own problem (13) is purely open-loop. However, Receding Horizon philosophy introduces feedback by stating that at the current time $t$ only first element of the optimal sequence is used

$$
S(t)=S_{t}^{*}(0)
$$

and at the next time step $t+1$ the optimal control problem (13) is solved over a shifted horizon (and with the new initial value of the sate vector).

The optimal control problem (13) can be recast as a Mixed Integer Quadratic Program. This can be accomplished via a Mixed Logic Dynamic (MLD) description of the system and elementary algebraic manipulation (see $^{18}$ for more details on the MLD formulation and the translation procedure). The translation to the MLD form can be done automatically with the program HYSDEL ${ }^{\ddagger}$.

It is well known that the class of mixed integer optimization problems is difficult. With the exception of some special cases, the time needed to solve such a problem grows exponentially with the problem size. For implementation we would like to obtain the solution in a state-feedback form $S(t)=f(x(t))$.

${ }^{\ddagger}$ HYSDEL (HYbrid System DEscription Language.) 2.0.0, see http://control.ee.ethz.ch/ hybrid/hysdel/hysdel.msql 


\begin{tabular}{|l|l|}
\hline Parameter & Value \\
\hline \hline$C_{m 1}$ & 1 \\
\hline$L_{m 1}$ & 0.0255 \\
\hline$R_{m 1}$ & 0.001 \\
\hline$C_{p}$ & 120 \\
\hline
\end{tabular}

\begin{tabular}{|l|l|l|}
\hline Type & $R$ & $L$ \\
\hline \hline$R$ Shunt & $1 \mu$ & - \\
\hline$R-L$ Shunt & $1 \mu$ & $21 \mu$ \\
\hline
\end{tabular}

Table 1. Parameters of the simulations

\section{SIMULATIONS}

In the following, we present some simulations for the switching $R$ and $R-L$ shunt using the Hybrid System Framework and compare the results with those of the Heuristic Controllers. The parameters of the model are summarized in Table 1. For simplicity, the model is normalized to a resonance frequency of $\omega_{n}=2 \pi \mathrm{rad} / \mathrm{s}$.

\subsection{Switching $R$-Shunt}

In this section, some results using the Receding Horizon Optimal Control (ROHC) technique for the switching $R$ shunt are presented. The number of possible switch state alterations within one period of the mechanical vibration is limited by the sample time $T_{s}$. As we are interested in an optimal switching sequence with as many possible optimal switch state changes, $T_{s}$ must be kept small. On the other hand, the optimization horizon should comprise several vibration periods in order to determine the damping of the structure. If this is not fulnilled, trivial solution will be obtained. Unfortunately, these two conditions contradict, because a long prediction horizon over many periods with small sample times results in a large number of steps for the prediction horizon and the calculation time will increase significantly. We chose $T_{s}=0.08$ and the prediction horizon $N_{s}=17$. The results of the simulations with the switching $R$ shunt are presented in Figure 4, where the switch is open for $S=0$ and closed for $S=1$. Figure 4 a) shows the heuristic State-Switching controller and b) the RHOC. The RHOC closes the switch only for a short time similar to the SSD-technique. This may be due to that fact, that the charge on the piezoelectric capacitance can be removed almost immediately, but it takes more time to recharge it again by the transducer. In Figure $4 \mathrm{c}$ ), the damping of the two switching laws is compared. It can be seen that the RHOC achieves better damping than the heuristic State Switching controller.

\subsubsection{Optimal Feedback Solution}

The receding horizon optimal control design in the last. section achieves a better vibration suppression than the heuristic State-Switching control law proposed in previous papers. However, the calculation time of the optimization problem for one single time step takes more than 3 seconds. As the sampling time of typical active vibration applications is less than one millisecond, the presented approach cannot be used in real time

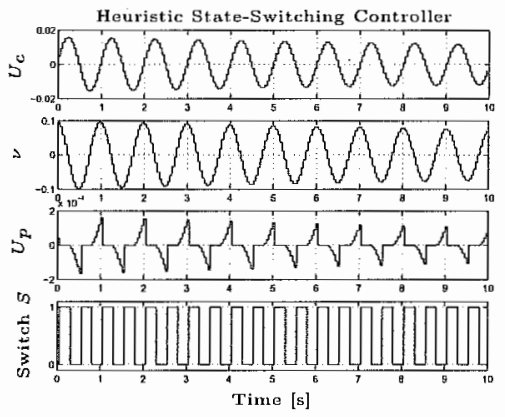

a)

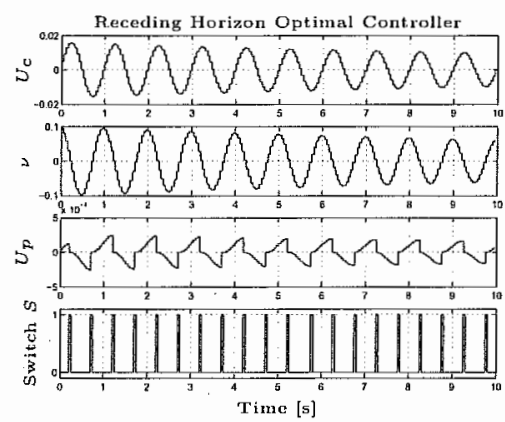

b)

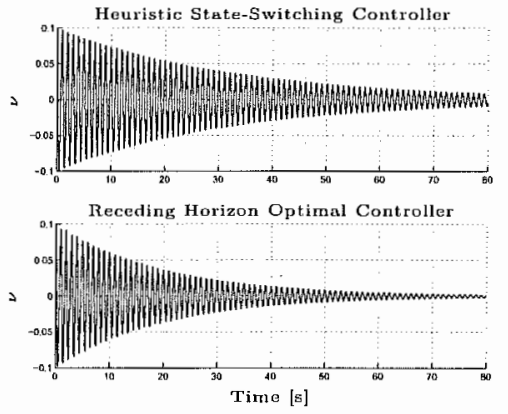

c)

Figure 4. Comparison between the two different switch controllers. a) Heuristic State-Switching Controller, b) Receding Horizon Optimal Controller with a prediction horizon of 17 steps, $T_{s}=0.08$ and $K_{i j}=\sqrt{C_{m i} / C_{p}}=0.0931$. 


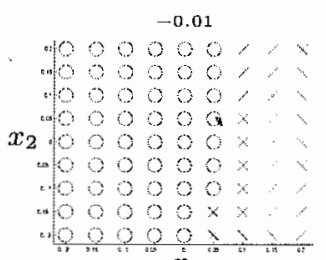

$x_{1}$
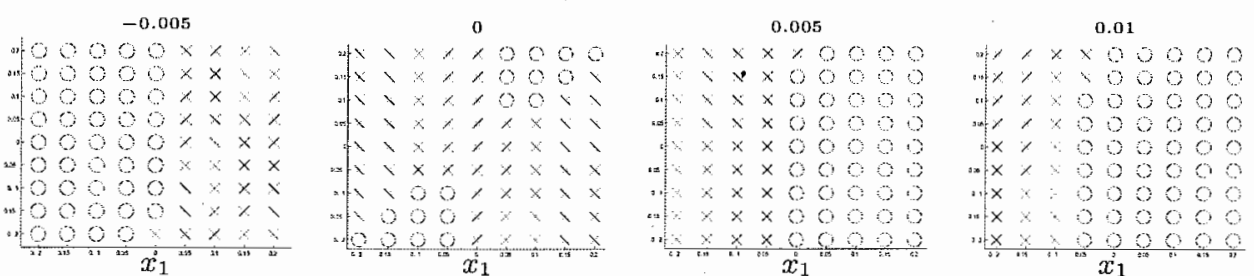

Figure 5. X-axis: $x_{1}=[-0.2,0.2]$, Y-axis: $x_{2}=[-0.2,0.2]$ for $x_{3}=[-0.01,-0.005,0,0.005,0.01]$. (Circle): $S=1$, (Cross): $S=0$

applications. Thus, we will construct an optimal feedback solution $S^{*}(t)=f(x(t))$ based on the receding horizon optimal control design and present a look-up table for the optimal switch states that can be used for real-time applications.

The look-up table is generated by plotting the optimal $S^{*}$ for a set of initial states like in Figure 5 , where the optimal $S^{*}$ are plotted as a function of the system states $x_{1}, x_{2}$ and $x_{3}$, corresponding to $U_{C}, \nu$ and $U_{P}$ in Equation (2). One can clearly see that there are regions where $S^{*}$ is either one or zero. The approximation of these regions can be stored in a look-up table and afterwards one just has to check in which region the actual state is and then apply the corresponding $S^{*}$. It would be even better, if approximate rules can be formulated on the basis of these regions in order to derive a switching law.

From the analytical model and in simulations, it can be observed that there is a strong correlation between $x_{2}$ and $x_{3}$, i.e. $x_{2} \sim-x_{3}$. If this correlation is considered, the approximated regions plotted in Figure 6 a) can be obtained. These regions are independent on $x_{3}$. These approximated regions are nothing but the State-Switching controller, because the switching law defined by these regions can be formulated as

$$
S(t)=\left\{\begin{array}{lll}
1 & \text { if } & x_{1}(t) \cdot x_{2}(t) \geq 0 \\
0 & \text { if } & x_{1}(t) \cdot x_{2}(t)<0
\end{array}\right.
$$

where $x_{2} \sim \dot{x}_{1}$. One can see that this is the same switching law as that of the State-Switching in Equation (9).

Of course, there are better approximation of the look-up table than that in Figure 6 a). One approximation that takes all three state-space variables into account is shown in Figure 6 b). It contains two polyhedra and therefore it is very easy to check if the current state is in one of the regions and then to apply the corresponding switching action $S^{*}$. In simulations, the controller defined by these regions was compared with the State Switching controller. It turned out that the new controller defined by the approximated regions performed slightly better than the State Switching controller, however in experiments, no improvement could be measured.

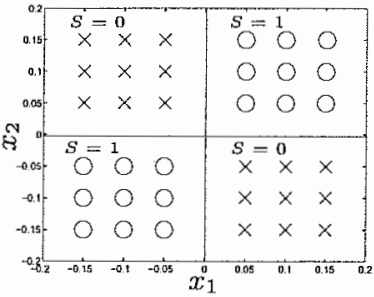

a)

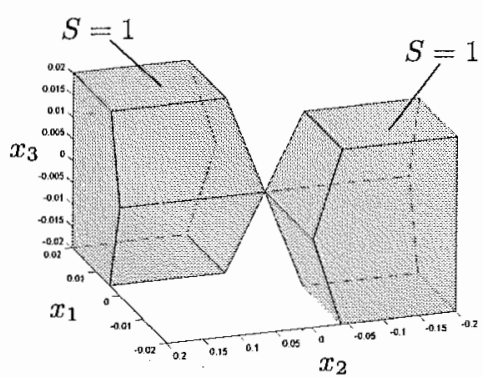

b)

Figure 6. a) Approximated look-up table, if the weak correlation $x_{2}=\nu \sim-x_{3}$ is considered. This look-up table corresponds to the ad hoc controller. b) Approximation of the regions taking into account all internal states of $G$. 


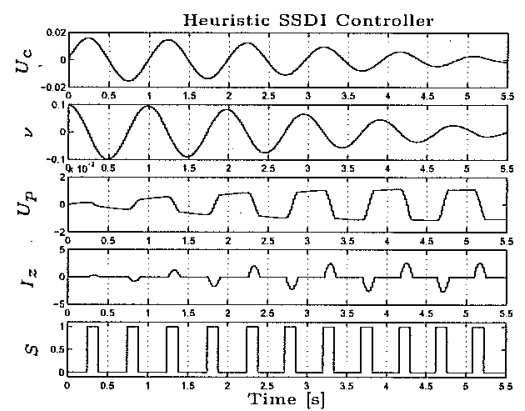

a)

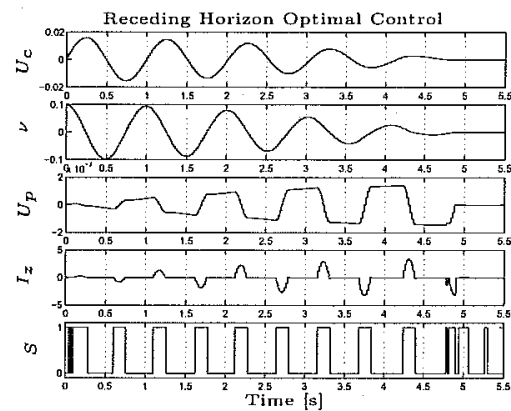

b)

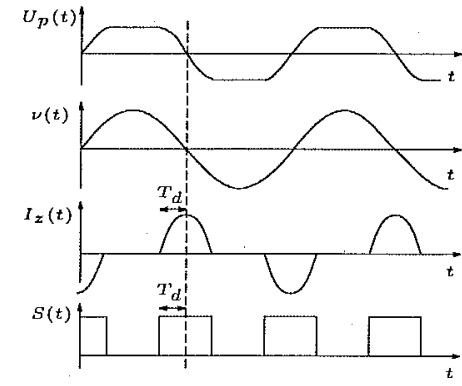

c)

Figure 7. Heuristic SSDI versus Receding Horizon Optimal Control with $T_{s}=0.02 \mathrm{~s}$. a) Heuristic Controller, b) Receding Horizon Optimal Control with Prediction Horizon of 22 . c) The switch is turned on $T_{d}=\pi \sqrt{L C_{p}} / 2$ before the product of the velocity $\nu(t)$ and $U_{z}$ becomes greater than zero according to the RHOC.

\subsection{Switching $R-L$ Shunt}

The problems of the Receding Horizon Optimal Control Design of the Switching $R-L$ shunt are similar to that of the switching $R$ shunt. Additionally, the order of the whole system is higher because of the inductor $L$ in the shunt. Therefore, one would expect that the calculation time of the RHOC takes more time. However, as the switching $R-L$ shunt can add more damping than the switching $R$ shunt, the problem is better conditioned and the calculation time of the optimization problem turns out to be smaller. The parameters of the model and the RHOC problem are shown in Table 1. In Figure 7 a) and b) the results are presented for the Heuristic Controller (SSDI-technique) and the Receding Horizon Optimal Controller. One can see that the RHOC turns on the switch earlier than the Heuristic Controller. Precisely, the switch is turned on $T_{d}=\pi \sqrt{L C_{p}} / 2$ before the product of the velocity $\nu(t)$ and $U_{z}$ becomes greater than zero (Figure $7 \mathrm{c}$ ). This means that the maximal current occurs exactly when the product of the velocity $\nu(t)$ and $V_{z}$ becomes greater than zero and therefore the damping of RHOC is slightly better than that of the heuristic SSDI controller. This is also shown in Figure 8 a), where the 2-norm of the velocity is plotted as a function of $L$ and the time when switching occurs. From this figure, it can be seen that optimal damping appears, when the switch is closed $T_{d}=\pi \sqrt{L C_{p}} / 2$ before the product of the velocity $\nu(t)$ and $V_{z}$ becomes greater than zero. It can be seen from these simulations, that an approximate switching law can be derived. This law is expressed as

$$
S(t)=\left\{\begin{array}{lll}
1 & \text { if } \quad t \geq T_{i}-\frac{\pi \sqrt{L C_{p}}}{2} \wedge I_{z} \neq 0 \\
0 & \text { if else }
\end{array}\right.
$$

with $T_{i}$ defined by $U_{p}\left(T_{i}\right) \cdot \nu\left(T_{i}\right)=0$.

Similar to the switching $R$ shunt, the optimal parameters $R$ and $L$ can be obtained by plotting the 2-norm of the velocity $\nu$ as a function of the parameters or a gradient search algorithm could be used to get the optimal values. We want to show the sensitivity of the damping when these parameters change and compare it to the traditional $R-L$ shunt. The results are plotted in Figure $8 \mathrm{~b}$ ) and c). As can be seen, the switching $R-L$ shunt is much more robust for varying parameters than the standard $R-L$ shunt. This is a great advantage of this shunt as the choice of parameters is not critical and the damping remains the same for environmental variations. Also, the switching $R-L$ shunt adapts automatically to frequency changes of the excitation or structural resonances, because the switching is derived from the measured velocity. On the other hand, the traditional $R-L$ shunt can get easily de-tuned. In this case, the damping will decrease significantly. We can conclude that the vibration suppression of the switching $R-L$ shunt is more robust against varying system parameters than the traditional $R-L$ shunt. Moreover, it is easier to build the switching $R-L$ shunt, since the inductance can be kept about 20 times smaller than for the $R-L$ shunt and thus no virtual or synthetic inductor is required. 


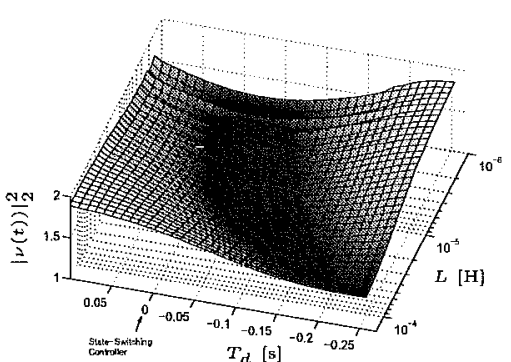

a)

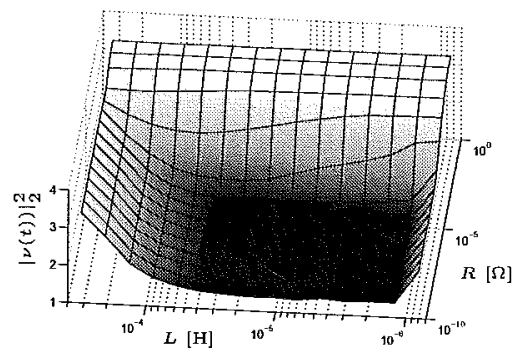

b)

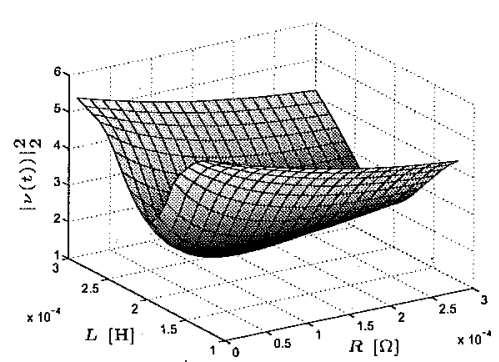

c)

Figure 8. a) 2-Norm of the velocity as a function of $L$ and $T_{\text {delay }}$, if the switching $R-L$ shunt is used. Optimal vibration suppression is obtained for $T_{\text {delay }}=\pi \sqrt{L C_{p}} / 2$. b) 2-Norm of the velocity as a function of $L$ and $R$, if the switching $R-L$ shunt is used. The vibration suppression is very robust for $L$ and $R$ variations. c) 2-Norm of the velocity as a function of $L$ and $R$ if a conventional $R-L$ shunt is used. The vibration suppression is very sensitive to $R$ and $L$ variations.

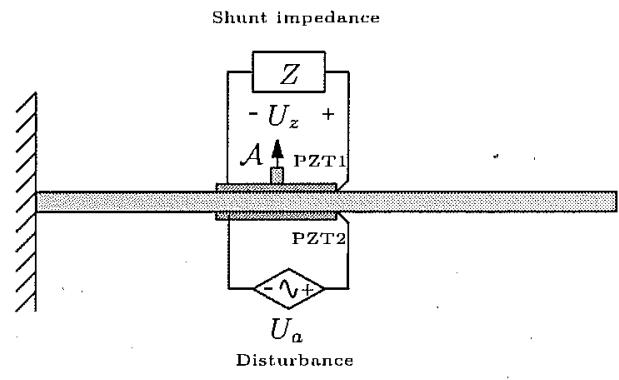

a)

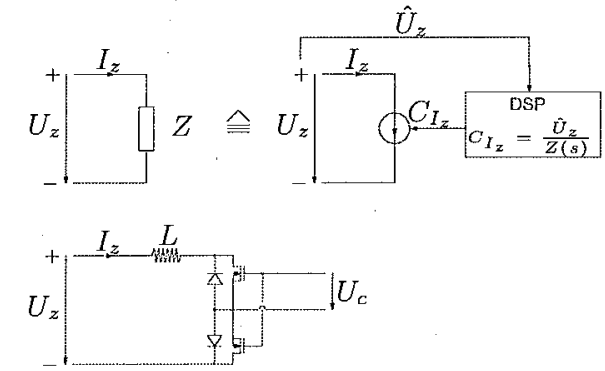

b)

Figure 9. a) Set-up of experimental piezoelectric laminated cantilever structure. b1) Synthetic impedance b2) Implementation of the switching $R-L$ shunt with solid state electronic components.

\section{EXPERIMENTS}

In this section, we present some experiments with the switching $R-L$ shunt on a cantilever beam.

\subsection{Set-up}

The experiments were carried out on a one-side clamped cantilever beam with two bonded piezoelectric patches (PZTs) as shown in Figure 9 a). One PZT patch is used as disturbance source and the other for shunt damping. The acceleration on the beam is measured with a collocated accelerometer $\mathcal{A}$. In order to implement the shunt impedance $Z$, the Synthetic Impedance Circuit ${ }^{21}$ shown in Figure 9 b1) is chosen. This type of impedance realizes the transfer function $U_{z} / I_{z}=Z$ by measuring the voltage $U_{z}$ and controlling the terminal current $I_{z}$. With this configuration, an arbitrary electrical network $Z$ can be implemented. Since the Digital Signal Processor, that controls the current source, is implemented with the XPC-Target System of MATLAB ${ }^{\S}$, the structure and parameters of the electrical shunt network can easily and quickly be changed. This accelerates the testing phase and allows parameter studies of the shunt damping system. Alternatively, the final implementation can be built with solid state electronic components in order to obtain a highly integrated and cheap product like in Figure 9 b2).

\footnotetext{
${ }^{\S}$ The MathWorks, Inc., 3 Apple Hill Drive, Natick, MA 01760-2098 USA, www.mathworks.com
} 


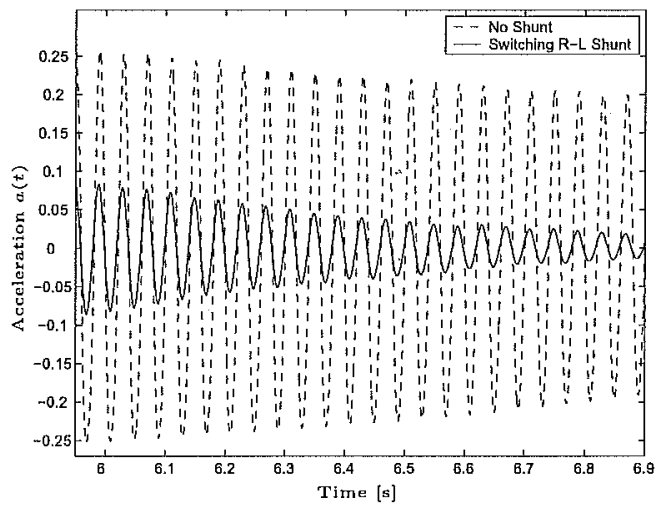

a)
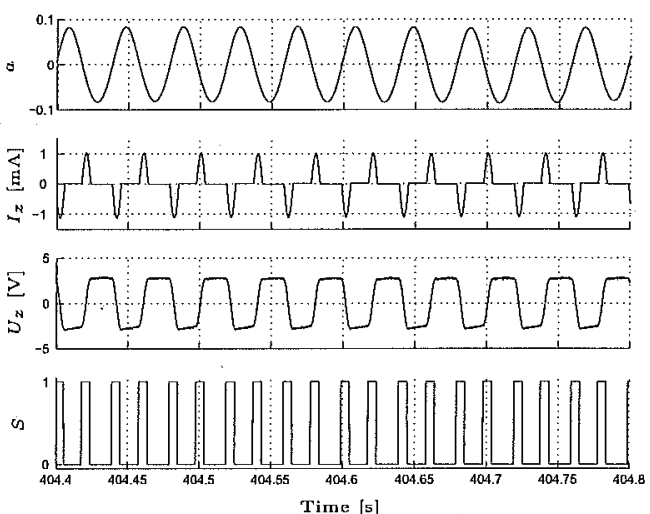

b)

Figure 10. Experimental results of the switching $R-L$ shunt where the 1st mode of the Beam is damped: a) Stepresponse of the acceleration a(t) for the undamped (dashed) and the switching $R-L$ shunt damped first mode vibration. b) Acceleration $a$, Current $I_{p}$, Voltage $U_{z}$ and switch state $S$.

\subsection{Results}

According to the simulations, the switching $R$ shunt does not add much damping to the structure. This was also observed in the experiment and therefore we will only present the experimental result for the switching $R-L$ shunt. For this purpose, the switching $R-L$ shunt is implemented on the synthetic impedance with $L=20 \mathrm{H}$. Figure 10 shows the experimental results for a tonal disturbance with the frequency of the first mode of the cantilever beam at $f=25 \mathrm{~Hz}$. One can see that the current peaks are around $1 \mathrm{~mA}$ and the shunt voltage is driven up to $3 \mathrm{~V}$ that counteracts with the vibration of the beam and results in a reduction of the acceleration by about $7 \mathrm{~dB}$. Figure $11 \mathrm{a}$ ) shows the 2-Norm of the acceleration for different switch-on times. As predicted by the Receding Horizon Optimal Control Design in Section 5.2, optimal vibration suppression appears when the switch is turned on before the velocity $\nu$ is zero. This is also shown in Figure 11 where the 2-norm of the acceleration is measured for different $L$ and $T_{d}$ values (b) and the acceleration as a function of time for different $T_{d}$ values (c).

\section{CONCLUSION}

In this paper, we introduced a new control design technique for switching shunted piezoelectric structures. The main motivation to develop this new design technique is that although recently proposed switching $R$ and $R-L$

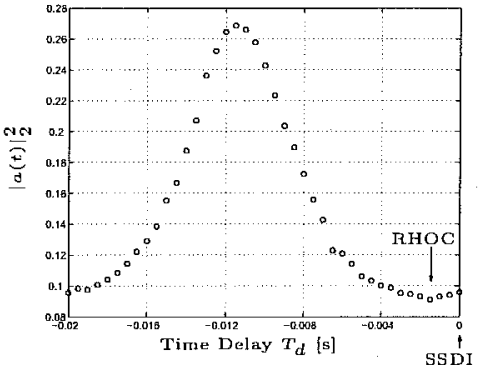

a)

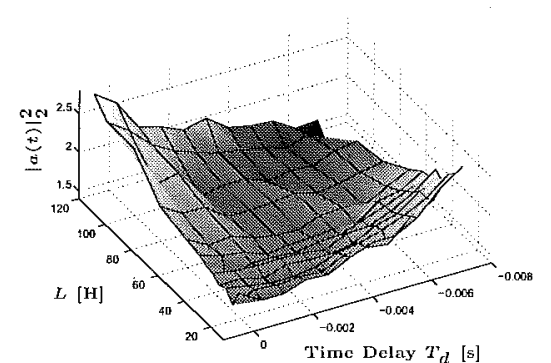

b)

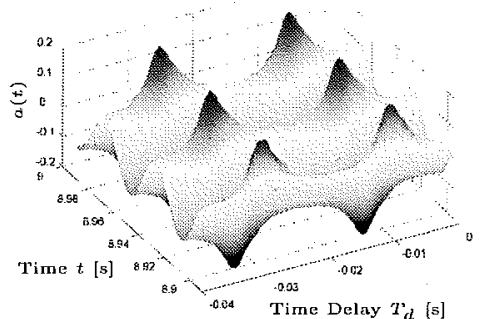

c)

Figure 11. Experimental results for the first mode with $f=25 \mathrm{~Hz}$ : a) 2-norm of the acceleration for different switch-on times $T_{d}$ b) 2-norm of the acceleration for different $L$ and $T_{d}$ values. c) Acceleration as a function of time for different $T_{d}$ 
shunt are easy to implement and can reduce structure vibration, the switching laws are derived heuristically. For this reason it remains unclear if a better switching law exists. We addressed this problem and demonstrated that the switching system can be modelled as a Hybrid System. Qnce the Hybrid System is obtained, a Receding Horizon Optimal Control Problem can be solved to obtain the optimal switching sequence. The Receding Horizon Optimal Controller could slightly improve the damping compared to the heuristically derived switching $R$ shunt controller. It was also shown that an approximation of the optimal feedback controller is the heuristic controller. For the switching $R-L$ shunt, the Receding Horizon Optimal Control design showed that the switching action should occur earlier than the heuristic controller implies. This improves the damping, which could also be demonstrated in experiments. We have shown that the Hybrid System Framework is a valuable tool to handle switching shunts and derive optimal controllers. This approach can be extended to more complex switching shunts (e.g. with more than one switch) in order to obtain optimal damping.

\section{ACKNOWLEDGMENTS}

Support for this research has been provided by a grant from ETH (Zurich) and EMPA (Dubendorf). The experimental facilities were provided by EMPA (Dubendorf) and the Synthetic Impedance Circuit was provided by the Laboratory for Dynamics and Control of Smart Structures (LDCSS). This work is part of a joint project between IFA ${ }^{\top}$, EMPAll $^{\|}$and IMES**. $^{* *}$

\section{REFERENCES}

1. C. R. Fuller, S. J. Elliott, and P. A. Nelson, Active Control of Vibration, Academic Press Limited, 24-28 Oval Road, London, 1996.

2. A. Preumont, Vibration Control of Active Structures - An Introduction, Kluwer Academic Publishers, Dordrecht, The Netherlands, 1991.

3. S. Elliott and P. Nelson, "Active noise control," IEEE-Signal-Processing-Magazine 10(4), pp. 12-35, 1993.

4. C. C. Sung and C. Y. Chiu, "Control of sound transmission through thin plate," Journal of Sound and Vibration 218(4), pp. 605-618, 1998.

5. N. W. Hagood and A. von Flotow, "Piezoelectric materials and passive electrical networks," Journal of Sound and Vibration 146(2), pp. 243-268, 1991.

6. J. Tang and K. W. Wang; "Active-passive hybrid piezoelectric networks for vibration control: comparisons and improvement," Smart Materials and Structures 10, pp. 794-806, 2001.

7. M. S. Tsai and K. W. Wang, "On the structural damping characteristics of active piezoelectric actuators with passive shunt," Journal of Sound and Vibration 221(1), pp. 1--22, 1999.

8. D. Niederberger, M. Morari, and S. Pietrzko, "Adaptive resonant shunted piezoelectric devices for vibration suppression," in Proc. SPIE Smart Structures and Materials - Smart Structures and Integrated Systems, Vol.5056, pp. 213-224, (San Diego, CA USA), March 2003.

9. A. J. Fleming and S. O. R. Moheinani, "Adaptive piezoelectric shunt damping," IOP Smart Materials and Structures 12, pp. 36-48, January 2003.

10. W. W. Clark, "Semi-active vibration control with piezoelectric materials as variable-stiffness actuators," in Proc. SPIE Smart Structures and Materials, Passive Damping and Isolation, SPIE Vol.3672, pp. 123-130, June 1999.

11. W. W. Clark, "Vibration control with state-switched piezoelectric materials," Journal of intelligent material systems and structures. 11, pp. 263-271, April 2000.

12. L. R. Corr and W. W. Clark, "Comparison of low-frequency piezoelectric switching shunt techniques for structural damping." IOP Smart Materials and Structures 11, pp. 370-376, 2002.

13. L. Corr and W. W. Clark, "A novel semi-active multi-modal vibration control law for a piezoelectric actuator," Journal of Vibration and Acoustics, Transactions on the ASME 125, pp. 214-222, April 2003.

\footnotetext{
"www.control.ethz.ch

${ }{ }_{\text {www.empa.ch/plugin/template/empa/57/ }}$

${ }^{* *}$ www.imes.ethz.ch/st/
} 
14. C. Richard, D. Guyomar, D. Audigier, and G. Ching, "Semi-passive damping using continuous switching of a piezoelectric device," in Proc. SPIE Smart Structures and Materials, Damping and Isolation, SPIE Vol.3672, pp. 104-111, April 1999.

15. C. Richard, D. Guyomar, D. Audigier, and H. Bassaler, "Enhanced semi-passive damping using continuous switching of a piezoelectric devices on an inductor.," in Proc. SPIE Smart Structures and Materials, Damping and Isolation, SPIE Vol.3989, pp. 288-299, (Newport Beach, CA), March 2000.

16. E. Sontag, "Interconnected automata and linear systems: A theoretical framework in discrete-time," in Hybrid Systems III - Verification and Control, R. Alur, T. Henzinger, and E. Sontag, eds., Lecture Notes in Computer Science(1066), pp. 436-448, Springer-Verlag, 1996.

17. A. Bemporad and M. Morari, "Control of systems integrating logic, dynamics, and constraints," 35, pp. 407427, Mar. 1999.

18. M. Morari, "Hybrid system analysis and control via mixed integer" optimization," in Proc. of the Sixth International Conference on Chemical Process Control (CPC-VI Proceedings), AIChE Symposium Series 326, 98, pp. 136-149, 2002.

19. F. Borrelli, Constrained Optimal Control Of Linear And Hybrid Systems, vol. 290 of Lecture Notes in Control and Information Sciences, Springer, 2003.

20. G. Larson, P. Rogers, and W. Munk, "State switched transducers: a new approach to high-power, lowfrequency, underwater projectors," Journal-of-the-Acoustical-Society-of-America 103, March 1998.

21. A. J. Fleming, S. Behrens, and S. O. R. Moheimani, "Synthetic impedance for implementation of piezoelectric shunt-damping circuits," IEE Electronics Letters 36, pp. 1525-1526, August 2000. 